\title{
Fast Atom Bombardment Tandem Mass Spectrometry Employing Ion-Molecule Reactions for the Differentiation of Phospholipid Classes
}

\author{
Mark J. Cole and Christie G. Enke \\ Department of Chemistry, Michigan State University, East Lansing, Michigan, USA
}

Fast atom bombardment tandem mass spectrometry, employing ion-molecule reactions with ethyl vinyl ether in a triple-quadrupole mass spectrometer, is used to differentiate classes of phospholipids. The phospholipids are desorbed and ionized by fast atom bombardment, mass-selected by the first quadrupole, and reacted with ethyl vinyl ether in the second quadrupole; the resulting product ions are analyzed by the third quadrupole. The protonated molecules and reaction product ions observed permit the differentiation of various phospholipid classes. The pattern of addition reaction products formed is shown to depend solely on the functionality of the lipid polar head group and not on the fatty acyl constituents. Neutral gain scans that are specific for each phospholipid class are performed. Ion dissociation products are observed in the same scan as the ion reaction products to provide data on the fatty acyl composition and position on the glycerophosphate core along with the phospholipid class. Although this method is less sensitive than neutral loss scanning for most phospholipid classes, it can (1) identify phospholipids that do not readily lose their head group as a neutral fragment and (2) detect phospholipids in mixtures containing species that give interfering neutral losses. (J Am Soc Mass Spectrom 1991, 2, $470-475)$

$\mathrm{T}$ he resurgence of interest in the mass spectrometry of phospholipids is due primarily to the development of fast atom bombardment (FAB) and tandem mass spectrometry (MS/MS). Fast atom bombardment allows the nonvolatile phospholipids to be analyzed without prior derivatization, and MS/MS allows phospholipid mixtures to be fully characterized without prior separation. Phospholipids are found in abundance in the cell walls and membranes of living organisms. Phospholipid research has recently centered around platelet-activating factor [1-3] and the use of phospholipids as biomarkers for the detection and identification of bacteria [4-6] and algae [7], and the determination of general membrane function in living matter.

The phospholipids in which we are presently interested are the glycerophospholipids (Structure I). Glycerophospholipids consist of four primary functional groups: a glycerol-3-phosphate core on which two fatty acyls ( $R$ and $R$ ) have been esterified to the two free hydroxyl groups in the sn-1 and sn-2 positions, and a second alcohol $(Y)$ esterified to the phosphate group in the sn-3 position. This head group $(Y)$

Address reprint requests to Christie G. Enke, Department of Chemistry, Michigan State University, East Lansing, MI 48824. is the functional group that determines the specific class to which the phospholipid belongs, whereas the fatty acyls distinguish the individual phospholipid species within each class. Examples of four different phospholipid classes are shown in Figure 1.

The usefulness of mass spectrometry in phospholipid analysis depends on the ability to detect and identify the phospholipids in complex matrices and mixtures. This requires a high degree of selectivity. For a method to be useful in obtaining a profile of phospholipids present in bacteria in natural samples, it must have high sensitivity as well as high selectivity. Although most of the phospholipid information is contained in the conventional FAB mass spectrum $[1$, 31, the data are difficult to interpret; lipid species at low concentrations are often buried in the high chemical background associated with FAB mass spectrometry, and FAB mass spectrometry does not work well for mixtures of phospholipids. Fast atom bombardment MS/MS analysis of daughter ions produced by collision-induced dissociation (CID) of molecular ion precursors has proven useful in phospholipid structure analysis [8-16]. Although this method is useful in obtaining information on the content, structures, and relative positions of the fatty acyls present on individual phospholipids, screening a mixture of 


\section{LIPID}

Phosphatidylethanolamine (PE) Phosphatidylglycerol
(PG)

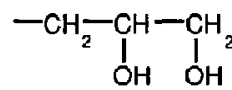

Phosphatidylcholine (PC)

Phosphatidylinositol (P!)
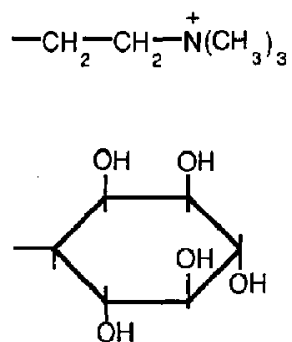

Figure 1. Examples of the head groups from four phospholipid classes.

phospholipids for the classes contained would be a complex procedure that requires a mathematical analysis of the daughter spectrum for every mass.

Constant neutral loss scanning for polar head functional groups has been shown to be very useful in the differentiation of phospholipid classes and analysis of phospholipids in complex matrices and mixtures [11, 17]. However, in low energy CID, some classes of phospholipids do not readily lose their polar head group as a neutral fragment, whereas others may share a neutral loss with an interfering species. Therefore, low energy CID may not always provide an unambiguous differentiation of the phospholipid classes contained in a complex mixture.

Several researchers have taken advantage of the

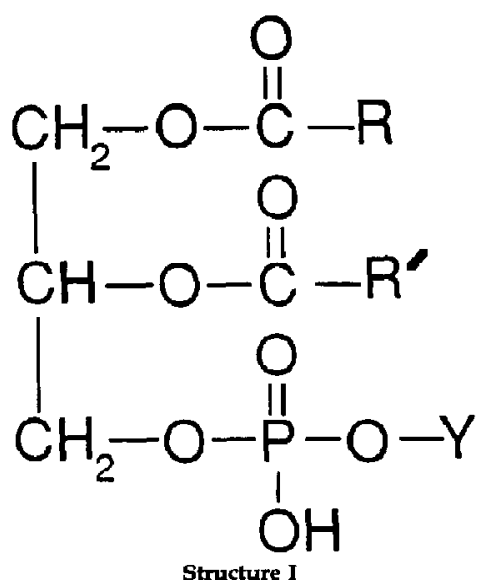

ability of the triple-quadrupole mass spectrometer to perform ion-molecule reactions to differentiate among closely related compounds [18-23]. In these applications, ion-molecule reactions were able to differentiate compounds that were difficult or impossible to differentiate by means of CID methods. In the present work, ion-molecule reactions of phospholipids with ethyl vinyl ether are explored as an alternative to low energy CID methods for the differentiation of phospholipid classes.

\section{Experimental}

All experiments were performed by using a triplestage quadrupole spectrometer (TSQ-70, FinniganMAT, San Jose, CA) equipped with a standard Finnigan FAB source and a saddle field gun (Ion Tech, Teddington, UK). Spectra were acquired and processed by using the Finnigan TSQ-70 data system and software.

All phospholipids were obtained from Sigma Chemical Co. (St. Louis, MO). Ethyl vinyl ether and glycerol were obtained from Aldrich Chemical Co. (Milwaukee, WI).

The phospholipid samples were dissolved in the glycerol matrix and 1-3 $\mu \mathrm{L}$ of this mixture was placed on the probe tip for analysis. Xenon was used as the FAB gas and the FAB gun was operated with a current flow of $2.4 \mathrm{~mA}$ and a beam energy of $7.5 \mathrm{keV}$. The individual protonated lipids formed in the ion source were selected by the first quadrupole $(Q 1)$ to pass through to the second quadrupole $(\mathrm{Q} 2$, radiofrequency only) for reaction. Ethyl vinyl ether was introduced into $Q 2$ to a pressure of 3.5-4.0 mtorr as indicated by the instrument's convectron gauge, which is connected to the reaction chamber. The reaction and dissociation products were analyzed by scanning the third quadrupole (Q3) over a wide mass range above and below the mass of the selected parent ion.

The formation and abundance of the reaction products depend on the instrumental parameters. The most important of these parameters are the dircet current offset on $Q 2$ and the voltages on the lenses immediately before and immediately after Q2. These potentials control the kinetic energy of the reactant ion being passed by $Q 1$. The instrumental parameters were optimized by adjusting them to maximize the abundance of a product ion of $\mathrm{m} / \mathrm{z} 101$ produced in Q2 when protonated glycerol ions react with ethyl vinyl ether. This ion is due to the loss of acetaldehyde from the complex of protonated and neutral ethyl vinyl ether molecules [18]. The optimum direct current offset on $Q 2$ was found always to be between +2.5 and $+4.0 \mathrm{~V}$; the lens immediately preceding Q2 was found to optimize between +5.0 and $+6.0 \mathrm{~V}$; the optimum voltage for the lens immediately following Q2 was found to be between -1.0 and $-5.0 \mathrm{~V}$. These 
conditions are optimal for the ethyl vinyl ether reactions used in this study.

\section{Results and Discussion}

\section{Unique Reaction Products}

Upon reaction with ethyl vinyl ether, each phospholipid class gave a unique pattern of reaction products that permitted the differentiation of the various phospholipid classes studied. Reaction product spectra from representative members of four different classes of phospholipids are shown in Figure 2. The selected reactant ion is the lowest mass peak in the spectrum. The masses of the neutral additions are given in parentheses above each product ion peak in the mass spectrum. Each phospholipid class except phosphatidylcholine gave at least one unique reaction product with ethyl vinyl ether. Phosphatidylcholine gave a nonunique reaction product, but it was the only class to give a single reaction product. The mech- anisms of the reactions that occur have not yet been deduced. However, the most probable compositions of product species in the observed addition reactions are listed in Table 1.

\section{Where the Reactions Occur}

For application in phospholipid class differentiation, only the reactions of ethyl vinyl ether with the polar head group will be useful. If the differences in reaction products were dependent on the differences in fatty acyl groups, then different reaction products would be obtained within the same class. The spectra of two phosphatidylethanolamine analogues, which differ only in their fatty acyl content, are shown in Figure 3. The same reaction products (relative to the reactant ion) were obtained in each spectrum. These data demonstrate that the reactions are not occurring on the fatty acyl moieties of the phospholipid, but are occurring on the polar head group. When fragment ions that have lost their polar head group are reacted,
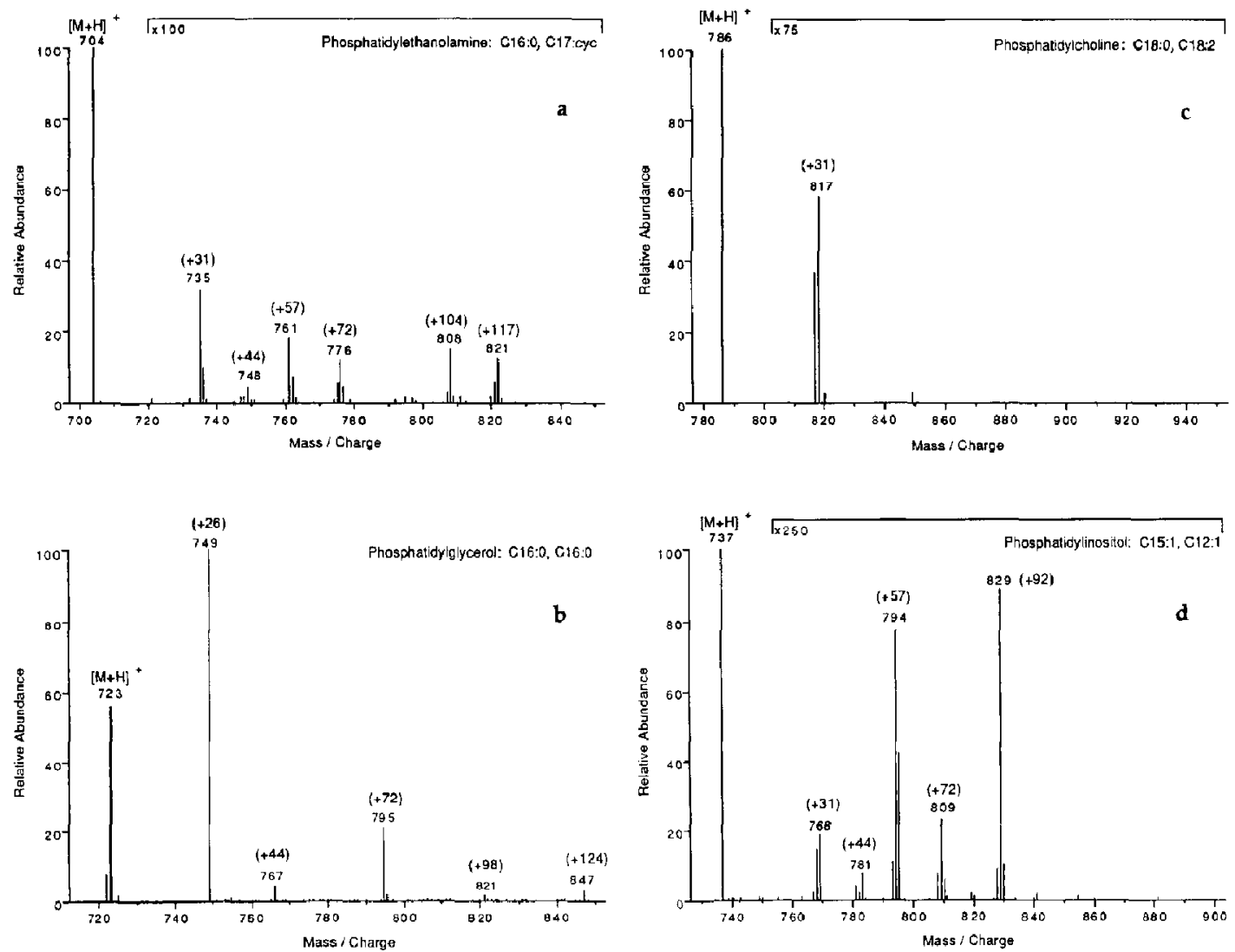

Figure 2. Mass spectra of the reactions of four different phospholipid classes. (a) PE C16:0, C17:cyc. (b) PG C16:0, C16:0. (c) PC C18:0, C18:2. (d) PI C15:1, C12:1, C16:0 is a 16-carbon fatty acyl with no unsaturation, C17:cyc is a 17-carbon propyl fatty acyl, C18:2 is an 18-carbon fatty acyl with two points of unsaturation, etc. The order in which the fatty acyls are listed correspond to their positions on the glycerol core. 
Table 1. Compositions of addition species

$\begin{array}{cc}\begin{array}{c}\text { Ethyl vinyl ether (Neu): } \mathrm{CH}_{2}=\mathrm{CH}-\mathrm{O}-\mathrm{CH}_{2}-\mathrm{CH}_{3} \\ \text { Mass }\end{array} & \begin{array}{c}\mathrm{C}_{2} \mathrm{H}_{2} \\ 26\end{array} \\ 31 & \mathrm{CH}_{3} \mathrm{O} \\ 44 & \mathrm{C}_{2} \mathrm{H}_{4} \mathrm{O} \\ 57 & \mathrm{C}_{3} \mathrm{H}_{5} \mathrm{O} \\ 72 & \mathrm{C}_{4} \mathrm{H}_{8} \mathrm{O}(\mathrm{Neu}) \\ 92 & 2 \mathrm{Neu}-\mathrm{C}_{4} \mathrm{H}_{8} \\ 98 & \mathrm{Neu}+\mathrm{C}_{2} \mathrm{H}_{2} \\ 104 & \mathrm{Neu}+\mathrm{CH}_{3} \mathrm{OH} \\ 117 & \mathrm{Neu}+\mathrm{C}_{2} \mathrm{H}_{5} \mathrm{O} \\ 124 & \mathrm{Neu}+\mathrm{C}_{4} \mathrm{H}_{8} \\ \end{array}$

all classes of phospholipids form the same reaction products. This is further evidence that the reactions are occurring on the polar head group and are thus indicative of the phospholipid class.

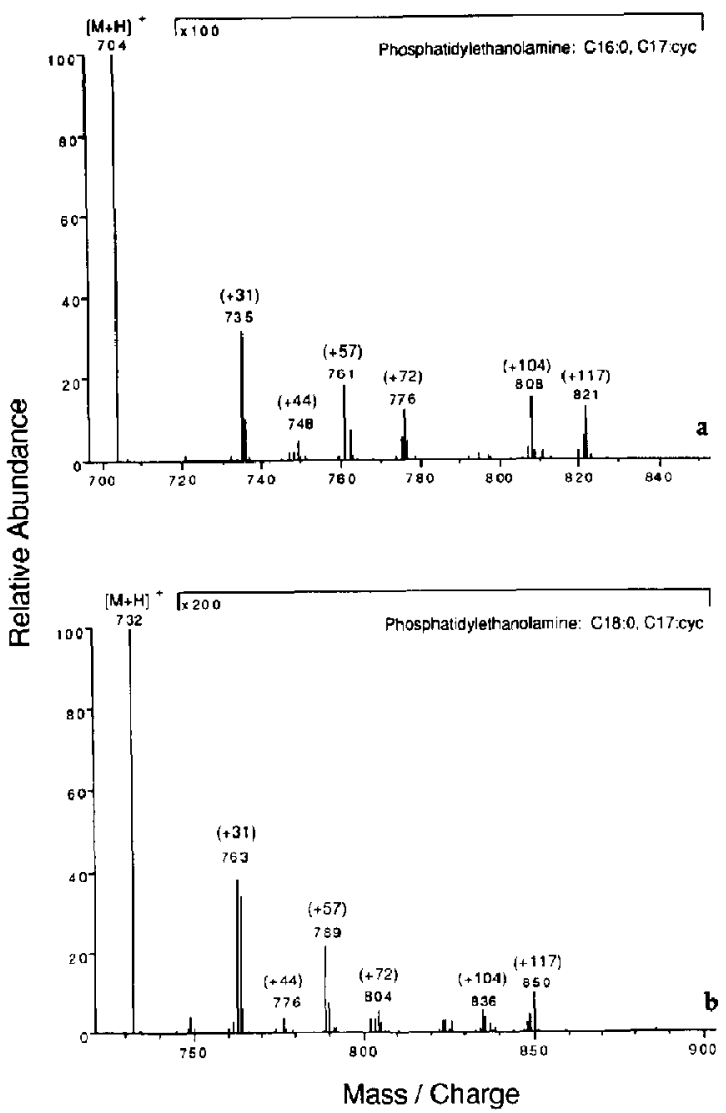

Figure 3. Mass spectra of two phosphatidylethanolamine analogues. These lipids are of the same class but differ in their fatty acyl content. (a) PE C16:0, C17:cyc. (b) PE C18:0, C17:cyc.

\section{Collision-induced Dissociation Data}

A full daughter scan of mass-to-charge ratio values above and below the mass value of the protonated molecule can provide both ion-molecule reaction data and CID data. A full daughter scan of a selected phosphatidyethanolamine parent ion that contains a 16-carbon saturated fatty acyl on the first position of the glycerophosphate core and a 17-carbon propyl fatty acyl on the second position is shown in Figure 4. In the positive ion mode, protonated phospholipids dissociate to form ions that contain information on their fatty acyl composition. These ions appear as the glycerol core with a loss of the head group and one or the other of the fatty acyls. The characteristic dissociation fragments shown in Figure 4 appear along with the characteristic reaction products. The peak corresponding to a loss of the fatty acyl from the sn-2 position is more intense than the peak corresponding to a loss of the fatty acyl from the sn-1 position. This conclusion is drawn from observations of the fragmentation patterns of different phospholipid classes containing various fatty acids. The same pattern is also observed in the conventional FAB mass spectrum of each phospholipid. These relative intensities observed in the positive ion spectra are reversed from those found by Jensen et al. [23] in the negative ion spectra, where the relative daughter ion peak intensity for the fatty acyl in the sn-2 position is greater than the daughter ion peak intensity for the fatty acyl in the sn-1 position. Huang et al. [24] have shown that this general rule apparently does not apply to phospholipids containing highly unsaturated fatty acyls or when large differences in the chain lengths of the two fatty acyls occur. It is not yet known whether these exceptions also occur in the positive CID spectra; this should be kept in mind when analyzing these types of data. However, for phospholipids that do not

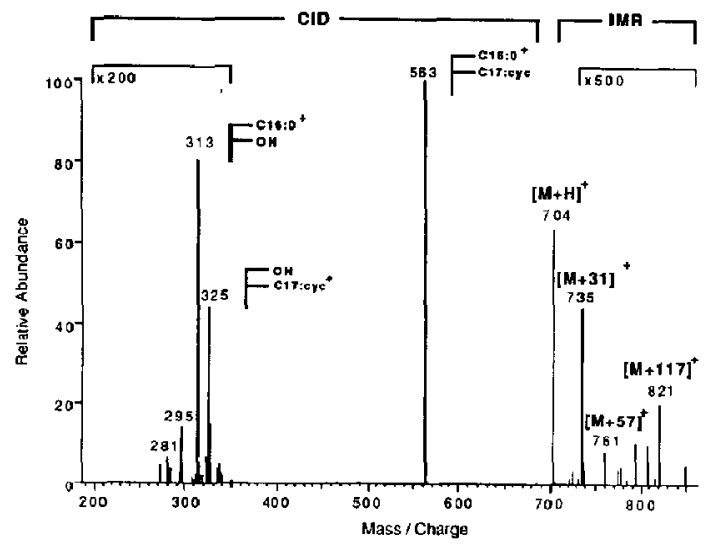

Figure 4. Full scan of a selected phosphatidylethanolamine parent showing collison-induced dissociation (CID) fragments as well as the characteristic reaction products from ion-molecule reactions (IMR). 
fall under these exceptions, a single scan can structurally characterize a phospholipid by giving the class of the phospholipid, the fatty acyls contained on the phospholipid, and the relative positions of the fatty acyls on the phospholipid.

\section{Neutral Gain Scans}

In addition to identifying classes of phospholipids through the unique reaction products of selected individual peaks in the mass spectrum, specific neutral gain scans that are selective for each particular class can be performed. In this way, all peaks in a mass spectrum that belong to a particular class are identified in the same scan. An example of a neutral gain scan is shown in Figure 5. The top spectrum is the conventional FAB mass spectrum of a crude lipid extract containing phosphatidylglycerol. The bottom spectrum is the neutral gain scan for the addition of $26 \mathrm{u}$, an ethyne moiety. This addition is specific for phosphatidylglycerol. The neutral gain scan selected only that peak in the conventional mass spectrum resulting from the protonated molecule of the single

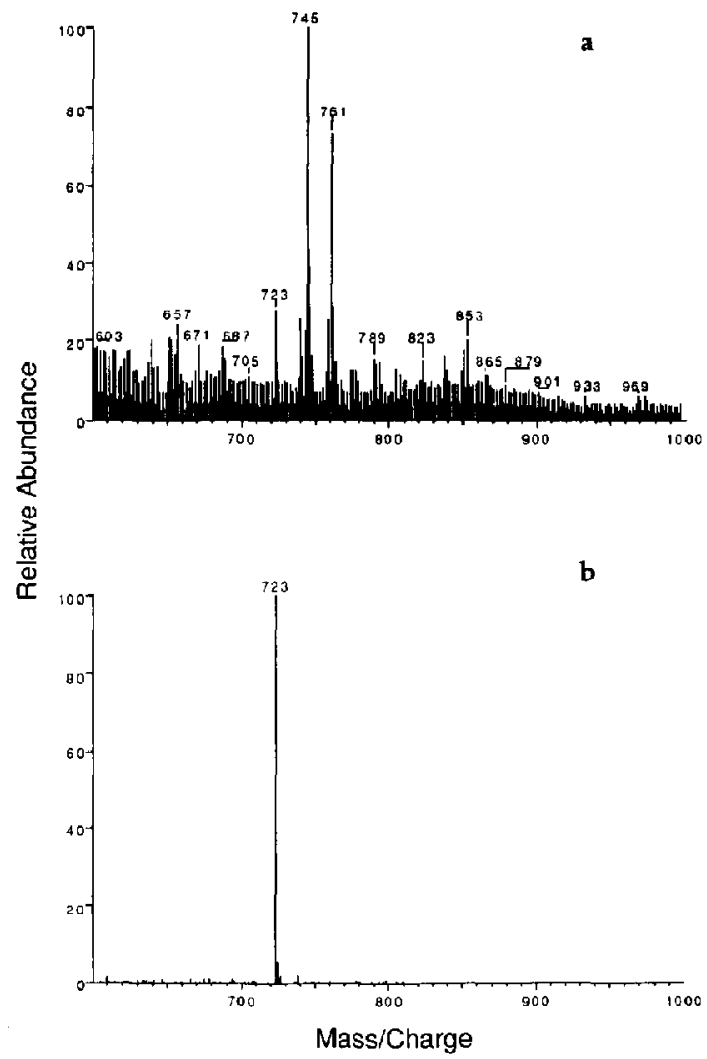

Figure 5. (a) Conventional mass spectrum of a sample containing phosphatidylglycerol. (b) Neutral gain scan for the addition of $26 \mathrm{u}$. This addition is specific for phosphatidylglycerol. phosphatidylglycerol species present. The neutral gain scan greatly increases specificity and signal-to-background ratio, thereby improving the detection limit. This technique is similar to neutral loss scanning with the advantage that those ions that do not fragment easily to lose their head group as a neutral loss do readily react to add a neutral gain.

A comparison of a neutral loss spectrum and a neutral gain spectrum is shown in Figure 6. The top spectrum is the neutral gain of $57 \mathrm{u}$, which is specific for phosphatidylinositol; the bottom spectrum is the neutral loss of $260 \mathrm{u}$ for the same sample. This neutral loss value also is specific for phosphatidylinositol. The actual intensity axis has been left on each spectrum for comparison. All of the phosphatidylinositol species present in the sample were detected by both scan modes. For phosphatidylinositol, the neutral loss spectrum is an order of magnitude more intense than the neutral gain spectrum. This fact is a function of the individual phospholipid class; the relative sensitivities of the two methods depend upon the facility with which each phospholipid class fragments to lose its polar head group as a neutral lnss. These inn-molecule reaction neutral gain scans do not provide information different from that obtained from neutral loss spectra, but they are highly advantageous in situations where neutral loss data are ambiguous or unavailable.
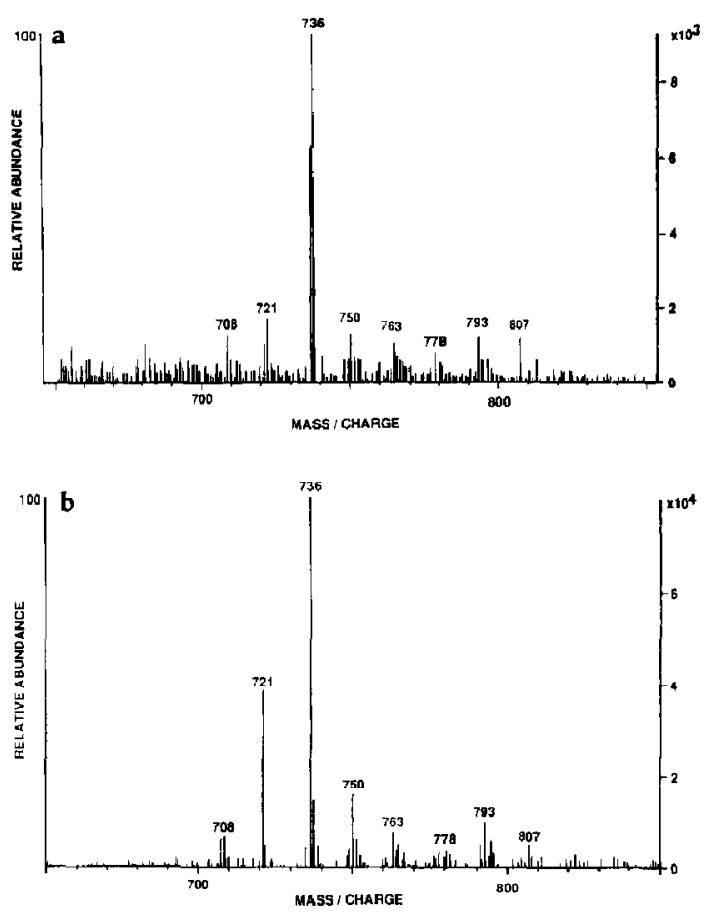

Figure 6. (a) Neutral gain of $57 \mathrm{u}$; specific for phosphatidylin nositol. (b) Neutral loss of $260 \mathrm{u}$; also specific for phosphatidylinositol. 


\section{Acknowledgments}

This work was supported by grants from the Michigan State University Research Excellence Fund and the National Science Foundation Center For Microbial Ecology at Michigan State University.

\section{References}

1. Plattner, R. D.; Stack, R. J.; Al-Hassan, J. M.; Summers, B.; Criddle, R. S. Org. Mass Spectrum. 1988, 23, 834-840.

2. Zirrolli, J. A.; Clay, K. L.; Murphy, R. C. Lipids, in press.

3. Weintraub, S. T.; Lear, C. S.; Pinckard, R. N. J. Lipid Res. 1990. 31, 719-725.

4. Heller, D. N.; Cotter, R. J.; Fenselau, C.; Uy, O. M. Anal. Chem. 1987, 59, 2806-2809.

5. Balkwill, D. L.; Leach, F. R.; Wilson, J. T.; McNabb, J. F.; White, D. C. Micrab. Ecol. 1988, 16, 73-84.

6. Pramanik, B. N.; Zechman, J. M.; Das, P. R.; Bartner, P. L. Biomed. Environ. Mass Spectrom. 1990, 19, 164-170.

7. Ross, M. M.; Neihof, R. A.; Campana, J. E. Anal. Chim. Acta 1986, 181, 149-157.

8. Sherman, W. R.; Ackerman, K. E.; Bateman, R. H.; Green, B. N.; Lewis, I. Biomed. Mass Spectrom. 1985, 12, 409-413.

9. Jensen, N. J.; Tomer, K. B.; Gross, M. L. Lipids 1986, 21 , $580-588$.

10. Jensen, N. J.; Tomer, K. B.; Gross, M. L. Lipids 1987, 22, 480-489.

11. Cole, M. J.; Enke, C. G. Presented at the 37th Annual
Conference on Mass Spectrometry and Allied Topics, Miami Beach, FL, May 21-26, 1989.

12. Hayashi, A.; Matsubara, T; Masanori, M.; Kinoshita, T.; Nakamura, T. J. Biochem. 1989, 106, 264-269.

13. Sweetman, B. J.; Tamura, M.; Higashimori, K.; Inagami, T.; Blair, I. A. Presented at the 35th Annual Conference on Mass Spectrometry and Allied Topics, Denver, CO, May 24-29, 1987.

14. Münster, H.; Budzikiewicz, H. Biol. Chem. Hoppe-Seyler 1988, 369, 303-308.

15. Kayganich, K. A.; Murphy, R. C. Presented at the 38th Annual Conference on Mass Spectrometry and Allied Topics, Tucson, AZ, June 4-9, 1990.

16. Easton, C.; Johnson, D. W.; Poulos, D. W. J. Lipid Res. 1988, 29, 109-112.

17. Heller, D. N.; Murphy, C. M.; Cotter, R. J.; Fenselau, C.; Uy, O. M. Antal. Chem. 1988, 60, 2787-2791.

18. Pachuta, R. R.; Kenttämaa, H. I.; Cooks, R. G.; Zennie, T. M.; Ping, C.; Chang, C.; Cassady, J. M. Org. Mass Spectrom. 1988, 23, 10-15.

19. Jalonen, J. I. Chem. Sac., Chem, Commun, 1985, 23, 872-874.

20. White, E. L.; Bursey, M. M. Biomed. Environ. Mass Spectrom. $1989,18,413-415$.

21. Meyerhoffer, W. J.; Bursey, M. M. Org. Mass Spectrom. 1989, 24, 169-175.

22. Kostiainen, R.; Auriola, S. Rapid Commun. Mass Spectrmm. 1988, 2, 135-137.

23. Jensen, N. J.; Tomer, K. B.; Gross, M. L. Lipids 1986, 21 , 580-588.

24. Huang, Z. H.; Gage, D. A.; Sweeley, C. C. I. Am. Soc. Mass Spectrom. 1990, in press. 